\title{
Modélisation de la morphodynamique des barres en croissant de la Côte aquitaine
}

\author{
Castelle, B., Bonneton, P. et Butel, R. \\ Département de Géologie et d'Océanographie, UMR CNRS 5805, Université Bordeaux I, 33405 Talence cedex
}

\section{Résumé}

Ce papier pressente la modélisation de la morphodynamique des plages sableuses d'Aquitaine où des bancs de sables complexes sont observes. Le modèle d'hydrodynamique sédimentaire MORPHODYN est couple avec le module de vagues spectral SWAN. Le couplage morphodynamique complet est effectue et nous permet de simuler la formation de barres sableuses par le mécanisme de "self-organization". Des barres en croissant sont formées dans la zone subtidale tandis que la mise en place d'un système barre/baïne est observé dans la zone intertidale. Les simulations montrent une forte modulation tidale des processus, ainsi que la sensibilité de la morphologie des croissants à la houle du large.

\section{Abstract:}

The present paper investigates the morphodynamics of the French Aquitan coast beaches which exhibit complex bed form features. The two-dimensional coastal area model MORPHODYN is coupled with the spectral wave driver SWAN. The entire morphological coupling allows us to form sand bar features from the self-organization mechanism. Simulations including tide cycles show the formation of crescentic bars in the subtidal zone and the formation of rip channels in the intertidal domain. Simulations show a strong tidal modulation of surf zone processes, and the crescent shape sensitivity to offshore wave conditions is revealed.

Mots-clés: barres en croissant, modélisation, auto-organisation, recirculations, rétroaction positive, morphodynamique.

\section{Introduction}

L'étude du comportement des bancs de sable de la côte aquitaine a été entreprise par le DGO depuis une dizaine d'années (voir par exemple Michel et al., 1994). Des barres en croissant sont observées dans la zone subtidale tout au long des $230 \mathrm{~km}$ de la Côte aquitaine (Froidefond ${ }_{3}^{2}$ et al., 1990), ainsi que le système barre/baïne dans la zone intertidale (De Melo Apoluceno et al., 2002.)

Le traitement d'images satellite SPOT et LANDSAT a permis récemment de déterminer certaines caractéristiques moyennes des barres en croissant (Lafon et al., 2004.).Sur la côte girondine ces barres ont une longueur d'onde moyenne comprise entre $500 \mathrm{~m}$ et $800 \mathrm{~m}$, mais des longueurs d'onde de plus en plus grandes peuvent être observées en se déplaçant vers le sud des plages landaises. 

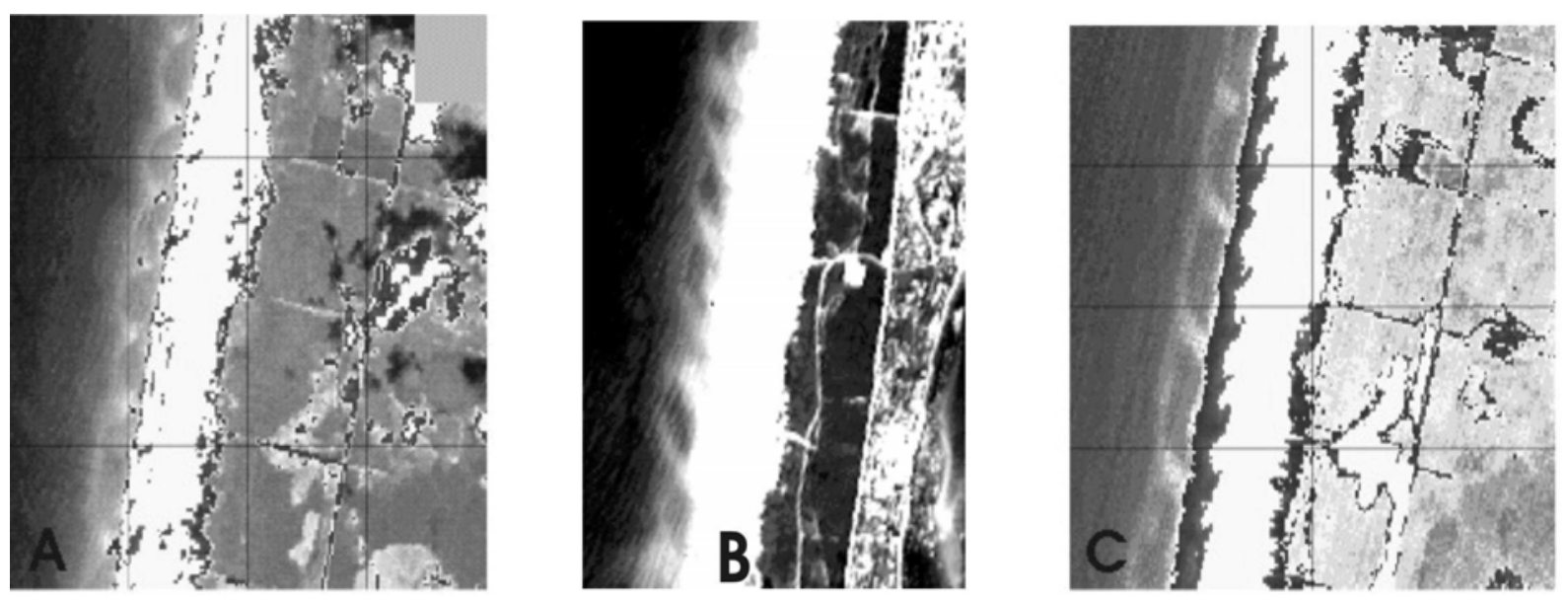

Figure.1: Images satellite des différentes formes de croissants subtidaux observes. A : SPOT@CNES, croissants symétriques ; B : LANDSAT@CNES, barres dissymetriques ; $:$ SPOT@CNES, barres "en lune"

La Figure 1 montre trois images satellite sur lesquelles on observe les différentes formes de barre en croissant. Elles peuvent être symétriques, dissymétriques, jusqu'à prendre la forme rarement observée de barres en lune orientées SE-NO. Le rôle que jouent ces barres est essentiel. En se déplaçant et en changeant de forme, les barres en croissant protègent la côte pendant les épisodes de grosses houles, en dissipant une grande partie de l'énergie des vagues au large. De plus ces barres stockent un grand volume de sédiment et contribuent ainsi fortement au budget sédimentaire littoral. Les barres en croissant sont aussi observées sur de nombreuses plages sableuses dans le monde, pourtant leur comportement morphodynamique reste un problème ouvert sur le plan international. C'est d'autant plus un défi pour la communauté scientifique que l'hydrodynamique sédimentaire côtière est l'objet de processus physiques fortement non-lineaire (vagues, courants et transport sédimentaire), voir chaotiques dans la zone de déferlement. Enfin, sur la Côte aquitaine, l'existence d'un marnage de l'ordre de $4.5 \mathrm{~m}$ en vive-eau induit une forte modulation tidale des processus dans la zone de surf ainsi qu'une interaction complexe entre les barres subtidales et intertidales (Castelle ${ }^{5}$ et al., 2004a.

Les deux principaux mécanismes physiques qui ont été évoqués afin d'expliquer la formation des barres en croissant sont : La théorie des ondes de bord ("edge wave theory"), et la théorie d'auto organisation ("self-organization theory").

Bowen et Inmann (1971) ont été les premiers à faire l'hypothèse que les ondes de bord stationnaires étaient responsables de la formation de ces barres en croissant, et ont ensuite étendu cette hypothèse à d'autres formes de bancs de sable (Holman and Bowen $\left.{ }_{9}^{7}, 1982\right)$. Cette théorie a été ensuite largement acceptée par la communauté (Carter , 1988 ; Komar , 1998 ; Short , 1999). Cependant il n'existe pas de preuve expérimentale que les ondes de bord soient à l'origine de la formation des barres en croissant : ne seraient-elles pas plutôt une consequence de leur presence ? De plus l'existence d'ondes de bord stationnaires peut paraître logique sur les plages délimitées par des pointes rocheuses ou des jetées, mais elle l'est beaucoup moins pour les plages qui s'étendent sur de longues distances (voir la discussion de Falques et al., 1996). Or les barres en croissant y sont largement observées : Aberdeen (Nova Scotia), Duck (Caroline du Nord), Palm Beach (Australie), plages d'Aquitaine et de Sete entre autres. 
Récemment, Reniers $^{12}$ et al. (2004) ont montré numériquement qu'il n'y avait pas de corrélation entre les ondes de bord forcées par les groupes d'onde et la longueur d'onde des barres, mais que ces structures rythmiques étaient le résultat de processus d'auto organisation. Ce mécanisme a été la première fois évoque par Sonu (1968) puis Barcillon et Lau (1973), et repris plus tard par Falquès et al. (1996). Il consiste en une rétroaction positive de l'hydrodynamique sur le fond sableux ("Bed-flow interation"), qui explique en particulier la formation de barres dans les rivières. Sur les plages exposées aux houles, l'évolution du fond va perturber le champ de houle incident ("Bed-surf interaction"). Si on considère un fond sableux auquel on superpose des perturbations aléatoires l'interaction non-lineaire entre l'évolution du fond et le champ de vague va conduire à la croissance de certaines perturbations jusqu'à la formation de bancs de sable.

Il existe deux approches permettant de simuler le mécanisme la "bed-surf interaction". La première est l'analyse de stabilité linéaire (voir par exemple Falquès et al., 2000 ; Calvette et al., 2001) qui permet de calculer la croissance initiale des instabilités, mais de ne considérer que des bancs de sable de faible amplitude. La deuxième consiste à suivre l'évolution du système non-lineaire entièrement couple (Coco et al., 2002, Damgaard et al., 2002). Les simulations aboutissent à la formation de barres en croissant et de barres transverses (Caballeria ${ }^{19}$ et al., 2002). C'est dans le cadre de cette dernière approche que s'inscrivent nos recherches.

L'étude discutée ici présente deux intérêts majeurs. Le premier intérêt est la prise en compte de la marée dans notre couplage morphodynamique ainsi d'étudier la modulation tidale des processus. Le deuxième intérêt est que les approximations couramment utilisées dans les précédentes études (houle monochromatique, transport sédimentaire simplifié...) sont ici écartées. Le couplage morphodynamique complet nous permet ainsi de suivre l'évolution de notre système non-lineaire et de mettre en évidence les processus physiques à l'origine de la formation des barres en croissant et de leur morphodynamique.

\section{Couplage morphodynamique}

MORPHODYN et SWAN ont été couples (Castelle ${ }^{20}$ et al. 2003) afin de pouvoir reproduire les courants induits par les houles aléatoires au-dessus de bathymétries complexes. Le module de houle SWAN résout l'équation de conservation de l'énergie de la houle aléatoire en milieu peu profond (Booij et al., 1999).

Le modèle MORPHODYN a été developpé par F. Saint-Cast ${ }^{22}$ (2002) dans le cadre d'une collaboration entre le DGO et le laboratoire MASTER. Il contient un module hydrodynamique et un module sédimentaire. L'hydrodynamique est résolue de manière implicite pour obtenir une solution quasi-stationnaire des débits liquides à partir des équations de conservation de la masse et de la quantité de mouvement. Les courants moyens Uc sont ensuite obtenus 'partir de la décomposition de Philipps (1977) qui permet de prendre en compte la contribution du courant de retour (Bonneton ${ }^{25}$, 2001), à laquelle on ajoute la contribution du roller d'après Kuriyama et al. (2000). Le couplage, le modèle mathématique utilise dans MORPHODYN et la nouvelle fermeture du modèle adoptée sont détailles dans Castelle et al. (2004b). La campagne de mesures PNEC 2001 (Sénéchal et al., 2004) a permit de collecter des données de vague et de 
courant pendant des conditions énergétiques et variées, et de calibrer le module hydrodynamique (Castelle et al., 2004a). Le module sédimentaire de MORPHODYN permet de calculer les flux sédimentaires à partir des courants moyens calcules au préalable. La formule de transport ici utilisée est celle de Bailard $^{28}$ (1981) qui prend en compte le transport sédimentaire par charriage, suspension et par effet de pente. Le nouveau fond est alors calculé à partir de l'équation de conservation du sédiment.

Le couplage morphodynamique complet avec prise en compte de la marée est effectue. La rétroaction du fond sableux sur la houle permet de prendre en compte le mécanisme de "Bed-surf interaction" suppose essentiel pour la formation des barres en croissant. Une pondération par la profondeur d'eau du transport sédimentaire par effet de pente est utilisée et nous permet d'effectuer des simulations de plusieurs semaines à plusieurs mois.

\section{Simulations morphodynamiques}

\subsection{Formation des barres en croissant}

Afin de parvenir à la formation de barres en croissant, nous avons considère comme situation initiale une plage présentant une barre subtidale rectiligne. Les conditions limitent latérales sont périodiques pour une plage de $3 \mathrm{~km}$ de long. Par conséquent, la longueur d'onde $\lambda \mathrm{b}$ des systèmes de barres formées lors des simulations est

$$
\lambda_{b}=\frac{3000}{n_{\text {croissants }}}
$$

où $\mathrm{n}$ croissants est le nombre de croissants formés sur la plage de $3 \mathrm{~km}$. Des perturbations aléatoires sont superposées à notre bathymétrie et le modèle morphodynamique couple calcule l'évolution de l'hydrodynamique sédimentaire et du fond sableux pour un nombre de cycles de marée donne et un pas de temps morpodynamique $\Delta$ tmorpho $=1$ heure. La figure 2 montre un exemple de simulation morphodynamique sur vingt jours en partant de la situation initiale précédemment d'écrite. La formation de petites recirculations de courants moyens développent certaines perturbations du fond. Le nouveau champ de vagues induit par ce changement du fond sableux va à nouveau induire la croissance de ces perturbations (rétroaction positive) et en lisser d'autres. Ces recirculations vont peu à peu s'organiser et être associées à la formation des barres en croissant.

Sur la Figure 2 après douze jours de simulation, on observe des barres en croissant dont la longueur d'onde $\Delta \mathrm{b}$ est de l'ordre de $600 \mathrm{~m}$, en accord avec les observations à partir d'images satellite. Dans la zone intertidale on observe la formation de chenaux ("rip channel"). Les simulations montrent que la formation de barres en croissant est observée pour les conditions de vagues au large suivantes :

-Houles énergétiques de hauteur significative Hs $>2.5 \mathrm{~m}$ environ. En effet, les houles peu énergétiques ne créent pas des courants moyens suffisamment intenses au-dessus de la barre subtidale pour développer les perturbations.

-Houle moyenne à longue : période entre $7 \mathrm{~s}$ et $16 \mathrm{~s}$ environ. Pour la taille des perturbations initiales considérées pour ces simulations, seules les houles moyennes ou longues vont réfracter suffisamment au-dessus de ces perturbations pour induire des recirculations de courants moyens capables de les développer.

-Houle frontale à quasi-frontale (incidence entre $0^{\circ}$ et $5^{\circ}$ environ). En effet, les houles trop obliques ne vont pas créer de recirculations de courants moyens au-dessus des perturbations. On observera plutôt un courant de dérive légèrement oscillant incapable de développer les perturbations. 


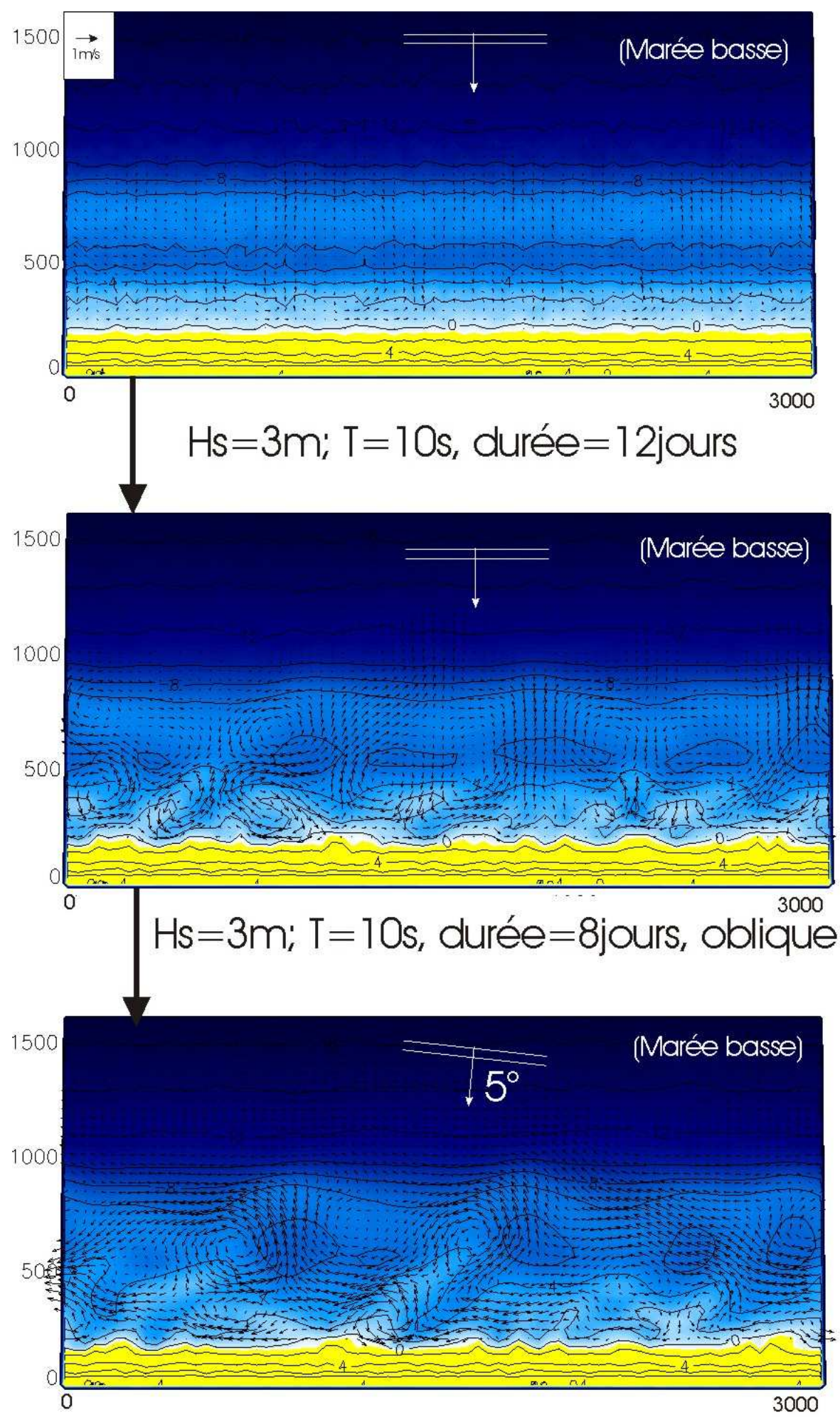

Figure. 2-Simulation morphodynamique. Formation de barres en croissant symètriques puis passage à une morphologie dissymétrique. Pas de temps morpho=1 heure, maillage regulier $75^{*} 34, \delta x=\delta y=40 \mathrm{~m}$ 


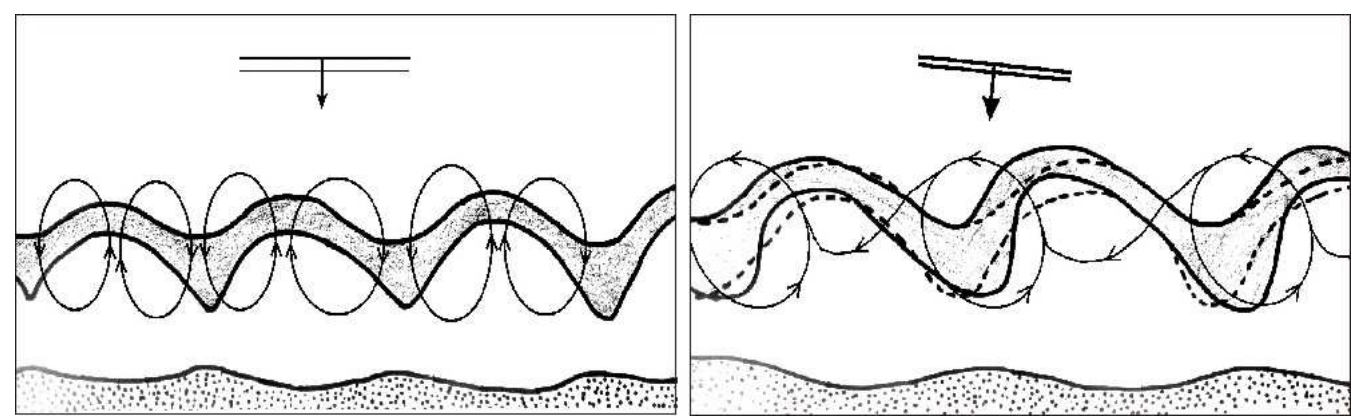

Figure. 3-Modèle de circulations de courants moyens associées aux barres en croissant symétriques et dissymétriques

\subsection{Morphologie des croissants}

Les houles frontales maintiennent les croissants, toutefois le système n'est pas en équilibre. On observe par exemple de forts échanges sédimentaires entre la zone subtidale et intertidale. Certains bancs se connectent alternativement aux systèmes intertidaux et subtidaux et sont la preuve d'une forte interaction morphodynamique entre les deux systèmes. Les points hauts des croissants peuvent légèrement migrer dans un sens ou dans l'autre dans la direction parallèlement à la côte, mais aucune migration du système dans sa globalité n'est observée. La modulation tidale de l'hydrodynamique sédimentaire dans les zones subtidale et intertidale est forte. En particulier, la dynamique des croissants est plus importante à marée basse.

Les houles énergétiques d'incidence obliques génèrent un courant de dérive intense. Dans la zone subtidale au-dessus des barres en croissant, une partie de ce courant alimente une recirculation de courant moyen. Pour une houle d'incidence NO cette recirculation est associée à un courant sagittal intense (ou "rip current") dirige vers le SO. Ceci contribue à une dissymetrisation du croissant en une durée de l'ordre de quelques cycles de marée (voir sur la Fig. 2 après huit jours de simulation). Le système migre lentement dans la direction du courant de dérive. La Figure 3 résume le processus auto organisation qui conduit à la formation de croissants symétriques ou dissymétriques pour les houles frontales ou obliques.

\section{Conclusion}

Les simulations montrent que la rétroaction positive du fond sableux sur le champ de houle est le processus physique qui conduit à la formation des barres en croissant. Cette formation est observée pour des houles moyennes à longues, quasi-frontales, et énergétiques. Lorsque ces barres en croissant existent, les houles énergétiques obliques vont leur faire prendre une forme dissymétrique. Ces formes ont déjà été observées sur des images SPOT ou LANDSAT. Les simulations nous montrent également la très forte modulation tidale des processus hydrosedimentaires qui agissent sur la Côte Aquitaine. Nos efforts doivent maintenant se porter sur le transport sédimentaire perpendiculaire à la côte et sur une quantification des mouvements de ces barres en croissant (longueur d'onde, vitesse de migration). A l'heure actuelle l'imagerie vidéo (du type ARGUS) semble le meilleur outil capable de les quantifier. 


\section{Références :}

1.Michel D. and Howa, H., (1994). Morphological evolution of a littoral sand bank, modelisation of its dynamics. Annals Geophysical, European Geophysical Union, Part II.

2.Froidefond J.-M., Gallissaires J.-M. and Prud'homme R., (1990). Spatial variation in sinusoidal on a crescentic nearshore bar: application to the Cap Ferret.Journal of Coastal Research, 6, 927-942

3.De Melo Apoluceno D., Howa H., Dupuis H., and Oggian G., (2002). Morphodynamics of Ridge and runnel systems during summer. J. of Coast. Res., SI 36, 222-230.

4.Lafon V., Dupuis H., Butel R., Castelle B., Michel D., Howa H., and De Melo Apoluceno D., (2004) Rhythmic subtidal bar morphology and dynamics in a mixed-energy environment: Part II: physical forcing analysis. Submitted to Estuarine Coastal and Shelf Science.

5.Castelle B., and Bonneton P., (2004a). Nearshore Waves and Current over Crescentic Bars. Apparaître dans J. of Coastal Res., SI 39, 2004a.

6.Bowen A.J. and Inman D.L., (1971). Edge waves and crescentic bars.J. of Geophysic. Res., $76,8662-8671$.

7.Holman R.A., and Bowen A.J., (1982). Bars, bumps and holes: models for the generation of complex beach topography. J. of Geophyic. Res., 87, (C1), 457-468.

8.Carter, R.W.G., (1988) Coastal Environments. Academic Press.

9.Komar P.D., (1998). Beach processes and sedimentation. Prentice Hall.

10.Short A.D., (1999). Handbook of Beach and Shoreface Morphodynamics. (Ed A.D. Short). Wiley

11.Falques A., Montoto A., and Iranzon V., (1996). Bed-flow instability of the longshore current. Cont. Shelf Res., 16, 1927-1964.

12.Reniers A.J.H.M., Roelvink J.A., and Thornton E.B., (2004). Morphodynamic mode-ling of an embayed beach under wave group forcing. Journal of Geophysical Research, 109, C01030, doi:10.1029/2002JC001586

13.Sonu C.J., (1968). Collective movement of sediment in littoral environment.Coast Eng., 337-400, Am. Soc. of Civ.

14. Barcillon A.I., and Lau J.P., (1973). A model for formation of transverse bars.J. of Geophys. Res., 78, 2656-2664.

15.Falqu'es A., Coco G., Huntley D.A., (2000). A mechanism for the generation of wave driven rhythmic patterns in the surf zone.J. Geophys. Res., 105 (C10), 24071-24088.

16.Calvette D., Falquès A., De Swart H.E. and Walgreen M., (2001). Modeling of the formation of shoreface-connected ridges on storm-dominated inner shelves. J. Fluid Mech., 218, $1-17$

17. Coco G., Caballeria M., Falquès A., and Huntley D.H., (2002). Crescentic bars and nearshore self-organization processes. In Proc. $28^{\text {th }}$ Int Conf. Coast. Eng., ASCE.

18.Damgaard J.S., Dodd N., Hall L., and Chesher T.J., (2002). Morphodynamic modelling of rip channel growth. Coast. Eng., 45, 199-221.

19.Caballeira M., Coco G., Falquès A., and Huntley D.A., (2003). Self-organization me chanisms for the formation of nearshore crescentic and transverse bars. J. Fluid Mech., 465, 379410.

20.Castelle B., Saint-Cast F., and Bonneton P., (2003). 2DH Modeling of sediment transport on a high energy barred-beach.Proceeding of Coastal Sediment '03, Florida. 
21.Booij N., Ris R.C., and Holthuijsen L.H., (1999). A third-generation wave model for coastal regions, Part I, Model description and validation.J. Geoph. Research, 104 (C4), 76497666.

22.Saint-Cast F., (2002). Modélisation de la morphodynamique des corps sableux en milieu littoral. Thèse de l'Université Bordeaux I, 246p, 2002.

23.Philipps O.M., (1977). The dynamics of the upper ocean. Cambridge University Press.

24.Bonneton P., (2001). Modélisation des courants générés par la houle en milieu littoral. Rapport interne DGO, 22p.

25.Kuriyama Y., Nakatsukasa T., (2000). A one-dimensional mode for undortow and longshore current on a barred beach. Coast Eng. 40, 39-58.

26. Castelle B., Bonneton P., Sénéchal N., Dupuis H., Butel R., and Michel, D., (2004b). Dynamics of wave-induced currents over a multiple barred-beach on the Aquitan coast. Submitted to Continental Shelf Research.

27.S'en'echal N., Bonneton P., and Dupuis H., (2001). Preliminary results of a field experiment on a barred beach, Truc Vert beach on October 2001. Ocean Dynamics, sous presse.

28.Bailard J.A., (1981). An energetics total load sediment transport model for a plane sloping beach. Journal of Geophysical Research, 86, (C11), 10938-10954, 1981 